\title{
Anestesia total intravenosa para tratamento endodôntico em Panthera leo - relato de caso
}

\section{Total intravenous anesthesia for endodontic treatment in Panthera leo - case report}

\author{
Larissa Colli Trebien ${ }^{1}$, Flávio Augusto Vieira Freitage², \\ Celina Tie Duque Nishimori ${ }^{3}$, Marcelo Bonat ${ }^{4}$, \\ Ricardo Guilherme D'Otaviano de Castro Vilani ${ }^{5}$
}

Resumo

\begin{abstract}
Animais presentes em zoológicos frequentemente necessitam de captura e anestesia para a realização de procedimentos clínicos e cirúrgicos. A anestesia total intravenosa apresenta vantagens como redução do estresse cirúrgico e menor depressão cardiovascular e respiratória. Entretanto, ainda são escassas as pesquisas dedicadas dentro deste contexto. Nesse sentido, o objetivo deste relato foi avaliar o protocolo anestésico empregado, visando um procedimento seguro e passível de reversão para a espécie silvestre selecionada. Acompanhou-se a anestesia de um leão (Panthera leo), macho, adulto, proveniente do Zoológico Municipal de Curitiba, submetido a procedimento endodôntico. A medicação pré-anestésica constituiu-se de dexmedetomidina $(6 \mu \mathrm{g} / \mathrm{kg})$, metadona $(0,2 \mathrm{mg} / \mathrm{kg})$, midazolam $(0,1 \mathrm{mg} / \mathrm{kg})$ e tiletamina-zolazepam $(1,2 \mathrm{mg} / \mathrm{kg})$. A indução foi realizada com propofol $(1,5 \mathrm{mg} / \mathrm{kg})$ e o animal foi intubado. Visando promover analgesia local, foi realizado bloqueio infraorbitário esquerdo com $5 \mathrm{~mL}$ de lidocaína a $2 \%$. A manutenção foi realizada por meio do fornecimento de propofol $(0,02-0,1 \mathrm{mg} / \mathrm{kg} / \mathrm{h})$, dexmedetomidina $(0,5 \mu \mathrm{g} / \mathrm{kg} / \mathrm{h})$ e remifentanil $(5 \mu \mathrm{g} /$ $\mathrm{kg} / \mathrm{h}$ ). O paciente apresentou sedação profunda e foi mantido em plano anestésico cirúrgico; todos os parâmetros fisiológicos monitorados permaneceram estáveis durante todo o procedimento. Após 55 minutos de anestesia o paciente apresentava sustentação espontânea da cabeça, quando foi novamente transportado ao zoológico para soltura no recinto. A ambulação foi considerada normal pelos observadores no zoológico seis horas após a anestesia. Pôde-se concluir que o protocolo realizado se mostrou tanto eficaz quanto seguro para a referida espécie encaminhada ao procedimento em questão.
\end{abstract}

Palavras-chave: Infusão contínua de propofol; Animais selvagens; Endodontia.

\footnotetext{
${ }^{1}$ Mestranda em Ciências Veterinárias (Medicina Experimental e Comparada) na Universidade Federal do Paraná (UFPR), Curitiba, Paraná, Brasil.E-mail: trebien.vet@gmail.com

${ }^{2}$ Doutorando em Ciências Veterinárias e Residência em Anestesiologia Veterinária (Ênfase em Anestesiologia Veterinária) na University of Guelph (U of G), Ontario Veterinary College (OVC), Guelph, Ontário, Canadá.

${ }^{3}$ Doutorado em Cirurgia Veterinária pela Universidade Estadual Paulista “Júlio de Mesquita Filho" (Unesp), Jaboticabal, São Paulo, Brasil. Professora Adjunta na Pontifícia Universidade Católica do Paraná (PUCPR), Curitiba, Paraná, Brasil.

${ }^{4}$ Graduação em Medicina Veterinária pela Universidade Federal do Paraná, Curitiba, Paraná, Brasil. Especialização em Clínica e Cirurgia de Animais Selvagens pela Faculdade Qualittas, Curitiba, Paraná, Brasil. Médico Veterinário, Chefe do Setor de Clínica do Zoológico Municipal de Curitiba, Curitiba, Paraná, Brasil.

${ }^{5}$ Doutorado em Ciências da Saúde pela Pontifícia Universidade Católica do Paraná, Curitiba, Paraná, Brasil. Professor Associado do Departamento de Medicina Veterinária da Universidade Federal do Paraná, Curitiba, Paraná, Brasil.
} 


\begin{abstract}
Zoo animals often require capture and anesthesia in order to undergo clinical and surgical procedures. Total intravenous anesthesia has advantages such as reduced surgical stress and less cardiovascular and respiratory depression. However, specific research on this matter is still scarce. Therefore, the present report aims to evaluate the anesthetic protocol employed, seeking a safe and reversible procedure for the selected wild species. A male adult lion (Panthera leo), from the Zoológico Municipal de Curitiba, was subjected to an endodontic anesthesia, under close monitoring. The pre-anesthetic medication consisted of dexmedetomidine $(6 \mu \mathrm{g} / \mathrm{kg})$, methadone $(0.2 \mathrm{mg} / \mathrm{kg})$, midazolam $(0.1 \mathrm{mg} / \mathrm{kg})$ and tiletamine-zolazepam $(12 \mathrm{mg} / \mathrm{kg})$. Induction was performed with propofol $(1 \mathrm{mg} / \mathrm{kg})$ and the animal was intubated. In order to promote local analgesia, a left infraorbital block was implemented with $5 \mathrm{~mL}$ of $2 \%$ lidocaine. Maintenance was undertaken by supplying propofol $(0.02-0.1 \mathrm{mg} / \mathrm{kg} / \mathrm{min})$, dexmedetomidine $(0.5 \mu \mathrm{g} / \mathrm{kg} / \mathrm{h})$ and remifentanil $(5 \mu \mathrm{g} / \mathrm{kg} / \mathrm{h})$. The patient exhibited deep sedation and followed the surgical anesthetic plan; all the monitored physiological parameters remained stable throughout the procedure. After 55 minutes of anesthesia the patient showed spontaneous head support, when it was transported back to the zoo. The ambulation was considered normal by the zoo observers 6 hours after the anesthesia. It was concluded that the protocol was both effective and safe for the referred species undergone the procedure reported.
\end{abstract}

Keywords: Propofol continuous infusion; Wild animals; Endodontics.

\section{Introdução}

Animais selvagens confinados em zoológicos e centros de triagem ou de pesquisa frequentemente necessitam de captura e anestesia para a realização de procedimentos clínicos e cirúrgicos de média à longa duração. ${ }^{(1)}$ Devido ao seu comportamento natural, especialmente a natureza agressiva, e risco à equipe, existe maior dificuldade na contenção desses animais. Além disso, esses animais têm maior suscetibilidade ao desenvolvimento de respostas fisiológicas exageradas em decorrência do estresse. No intuito de reduzir o estresse e facilitar o manejo, o condicionamento dos animais por meio do reforço positivo em zoológicos é uma valiosa ferramenta. Alguns dos benefícios incluem a redução do comportamento agressivo, facilitando a realização de alguns procedimentos veterinários menos invasivos ou a contenção desses animais. ${ }^{(2)}$

Independentemente do manejo empregado, o transporte entre instituições, de um leão, está associado com alterações fisiológicas em decorrência de estresse, seguido da liberação de catecolaminas, aumentando dessa forma o metabolismo celular e consequentemente o requerimento por anestésicos para captura e contenção. Destarte, é necessário cautela na escolha de fármacos que favoreçam a contenção e permitam uma anestesia seguras para a equipe e para o paciente. $^{(3)}$

Uma das técnicas de anestesia utilizadas nos dias de hoje, que apresenta um bom nível de segurança, é a anestesia intravenosa total (TIVA). Esta é uma técnica que tem sido difundida na prática anestésica em pequenos animais, especialmente motivada pela introdução de fármacos de ações mais curtas e rápidas, além do aperfeiçoamento das bombas de infusão. ${ }^{(4)}$ Induções suaves e curtos períodos de recuperação são características peculiares do propofol. ${ }^{(5)}$ Além disso, o propofol apresenta boa estabilidade hemodinâmica, poucos efeitos indesejáveis e ausência de efeito cumulativo para a maioria das espécies estudadas, ${ }^{(4)}$ o que o qualifica para ser utilizado em infusão contínua para manutenção anestésica. ${ }^{(5)}$

Entretanto, o propofol não possui propriedades analgésicas, e para que o protocolo de TIVA seja adequado é importante a adição de fármacos que apresentem propriedades analgésicas, como opioides e $\alpha$-2 agonistas. Dentre os opioides, o remifentanil, que é um agonista total de receptores $\mu$, 
trouxe novas perspectivas para o uso da TIVA, uma vez que apresenta rápido início de ação, meia vida contexto sensível sempre curta e potente analgesia, além de manter estabilidade hemodinâmica com mínimos efeitos na contratilidade cardíaca. ${ }^{(6-7)}$

Um ponto importante a ser ponderado, levando em consideração os gatos domésticos, é a dificuldade da espécie para glucuronidação e oxidação do propofol, podendo levar a um efeito cumulativo da droga, proporcionando clinicamente recuperações mais longas que nas demais espécies, especialmente quando a infusão é realizada por longos períodos. ${ }^{(8)}$ Infelizmente, a literatura na medicina veterinária traz um número restrito de informações quanto à utilização de TIVA em felídeos selvagens, em especial com uso de infusão de propofol por períodos longos, tornando relevante a publicação de pesquisas e relatos de caso que envolvam a TIVA nessas espécies. Desse modo, o objetivo deste manuscrito é relatar e avaliar a anestesia por um período de 150 minutos em um exemplar de leão africano (Panthera leo) utilizando infusão intravenosa de propofol, remifentanil e dexmedetomidina.

\section{Relato de caso}

Um leão africano (Panthera leo), macho, adulto, com idade estimada em 15 anos, pesando $176 \mathrm{~kg}$, foi encaminhado ao Hospital Veterinário da Universidade Federal do Paraná (HV-UFPR), município de Curitiba, estado do Paraná, Brasil, para tratamento endodôntico devido à fratura de canino. Previamente à pesagem e, portanto, à administração dos anestésicos para captura, estimouse um peso de $170 \mathrm{~kg}$ ao animal.

Sua captura foi realizada inicialmente no Zoológico Municipal de Curitiba através da introdução de uma caixa de madeira e grades em seu recinto, para que dessa maneira o animal se habituasse ao novo objeto. No dia do procedimento, por encontrar-se em jejum para a anestesia, foi facilmente atraído por seus tratadores para dentro desta caixa por meio da apresentação de pedaços de carne. Após fechar a caixa, foi transportado ao hospital e a contenção farmacológica para captura foi realizada à distância, por via intramuscular, mediante o disparo de um dardo anestésico, através de zarabatana, contendo dexmedetomidina $(6 \mu \mathrm{g} / \mathrm{kg})$, metadona $(0,2 \mathrm{mg} / \mathrm{kg})$, midazolam $(0,1 \mathrm{mg} / \mathrm{kg})$ e tiletamina-zolazepam $(1,2 \mathrm{mg} / \mathrm{kg})$.

Após 15 minutos da aplicação intramuscular, o animal foi levado ao centro cirúrgico de grandes animais e devidamente posicionado na mesa cirúrgica para a punção da veia cefálica esquerda com cateter de tamanho $18 \mathrm{G}$. A indução anestésica foi realizada com propofol na dose total de $1,5 \mathrm{mg} / \mathrm{kg}$, em bolus lento. Logo após, foram instilados $5 \mathrm{~mL}$ de lidocaína na glote e o animal foi intubado (vide Figuras 1 e 2) com traqueotubo de calibre $22 \mathrm{~mm}$, com auxílio de palpação das estruturas da laringe. $\mathrm{O}$ paciente foi mantido em ventilação mecânica controlada a volume, com um volume total de $2 \mathrm{~L}$, relação inspiração/expiração de 1:3, pressão expiratória final positiva (PEEP) de $2 \mathrm{mmHg}$ e com frequência respiratória inicial de quatro movimentos por minuto, com uso de oxigênio $100 \%$.

A anestesia foi mantida pela infusão contínua de propofol iniciando em $0,1 \mathrm{mg} / \mathrm{kg} / \mathrm{min}$, dexmedetomidina $(0,5 \mu \mathrm{g} / \mathrm{kg} / \mathrm{h})$ e remifentanil $(5 \mu \mathrm{g} /$ $\mathrm{kg} / \mathrm{h}$ ). A avaliação do plano anestésico foi feita com o objetivo de manter o paciente com o globo ocular rotacionado, sem reflexo palpebral lateral, sem tônus mandibular e movimentação de língua e sem reflexo anal. Foi permitido, porém, leve reflexo palpebral medial. Quando observada centralização parcial do globo ocular, sem reflexo palpebral lateral e sem tônus mandibular, a taxa de infusão do propofol era reduzida em $0,02 \mathrm{mg} / \mathrm{kg} / \mathrm{min}$. Dessa forma, após 40 minutos de anestesia, a taxa de infusão de propofol foi mantida em $0,02 \mathrm{mg} / \mathrm{kg} / \mathrm{min}$.

Visando ainda o controle da dor trans e pósoperatória, foi realizado bloqueio infraorbitário esquerdo com $5 \mathrm{~mL}$ de lidocaína a 2\% (Figura 3). 
Figura 1 - Paciente da espécie Panthera leo na mesa cirúrgica sendo preparado para intubação endotranqueal no Hospital Veterinário da Universidade Federal do Paraná (HV-UFPR) para tratamento endodôntico.

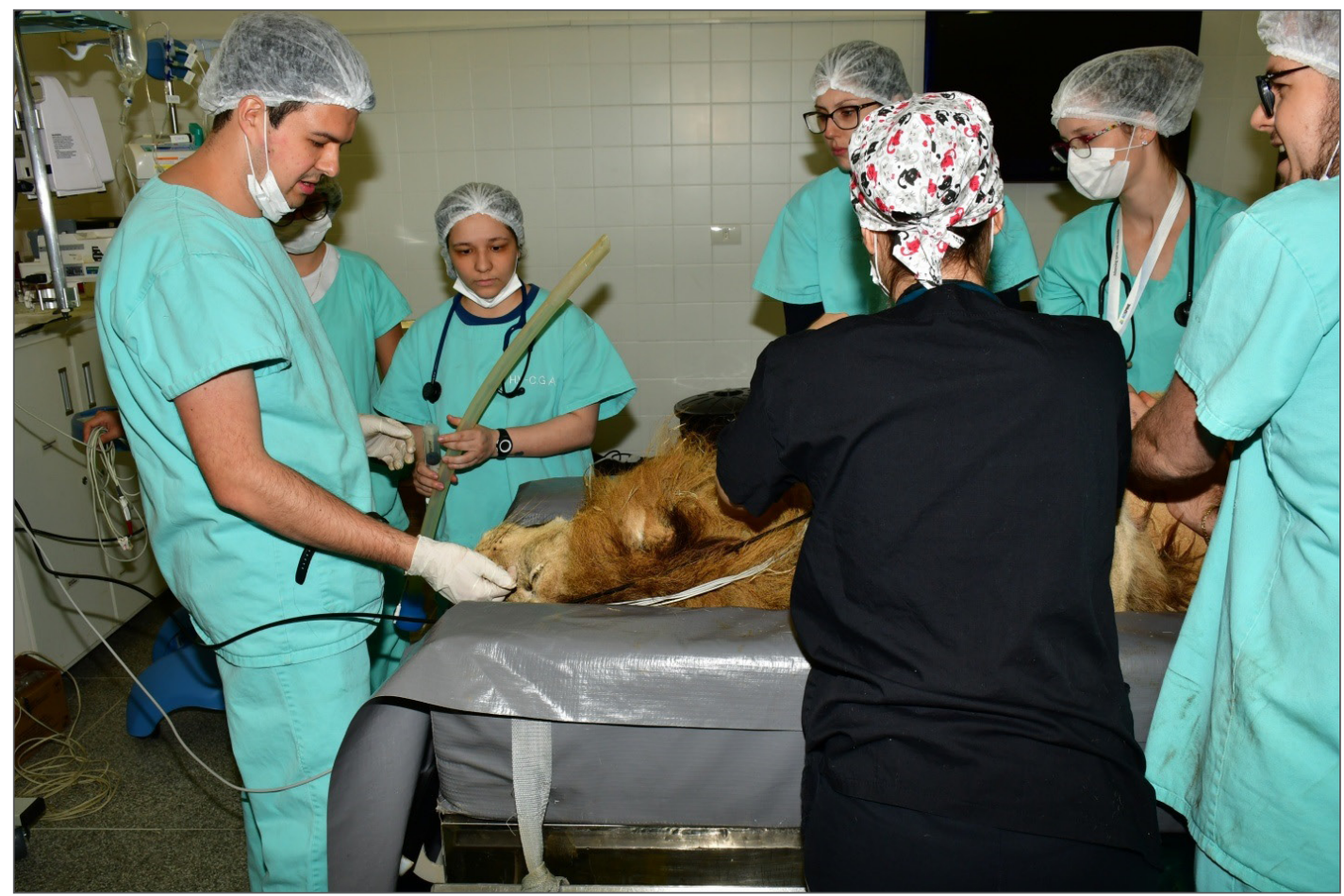

Fonte: os autores; fotografia cedida por Rogério Ribas Lange.

Figura 2 - Intubação endotraqueal em Panthera leo encaminhado para tratamento endodôntico no Hospital Veterinário da Universidade Federal do Paraná (HV-UFPR).

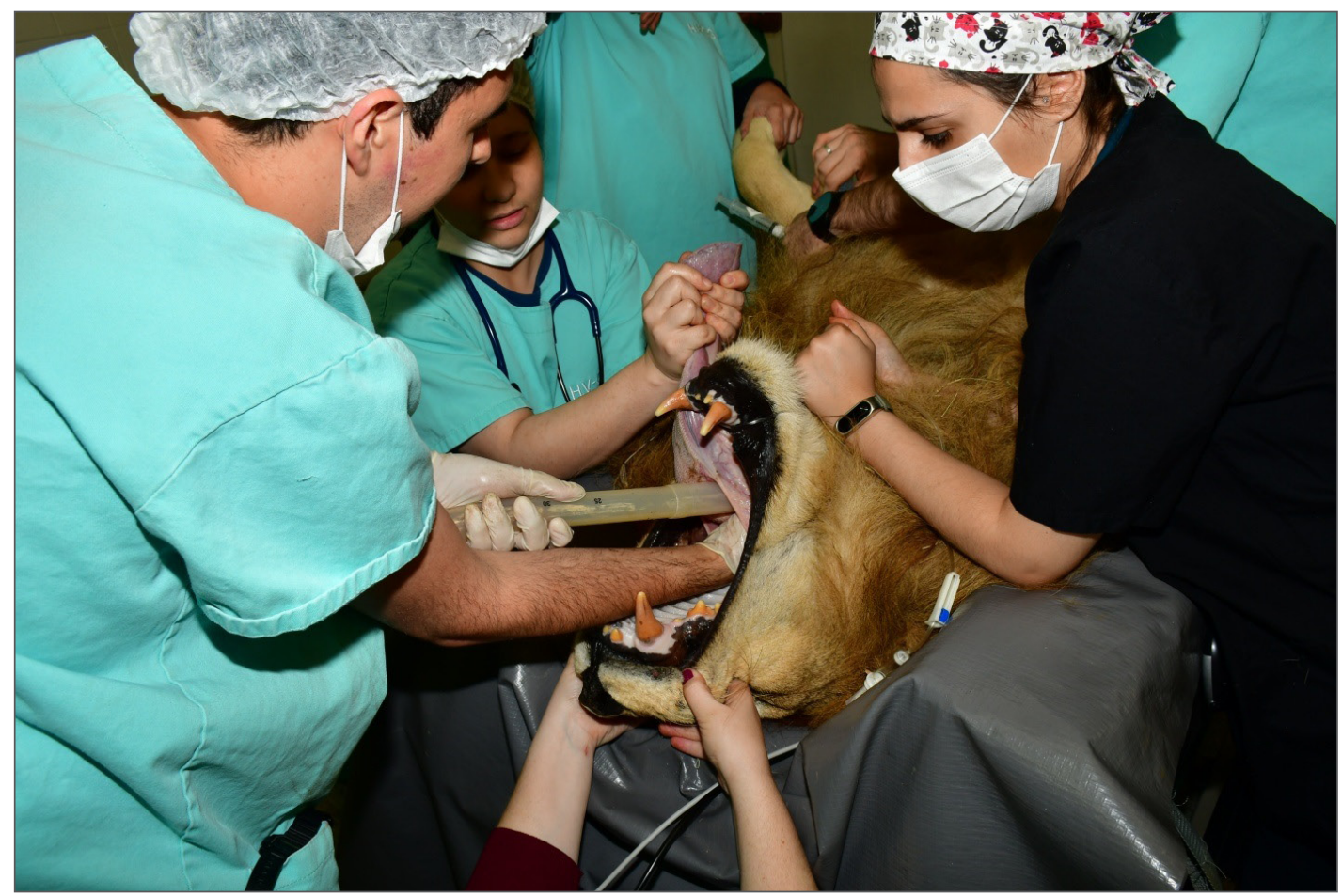

Fonte: os autores; fotografia cedida por Rogério Ribas Lange. 
Figura 3 - Bloqueio local infraorbitário esquerdo com $5 \mathrm{~mL}$ de lidocaína a $2 \%$ em paciente da espécie Panthera leo no Hospital Veterinário da Universidade Federal do Paraná (HV-UFPR).

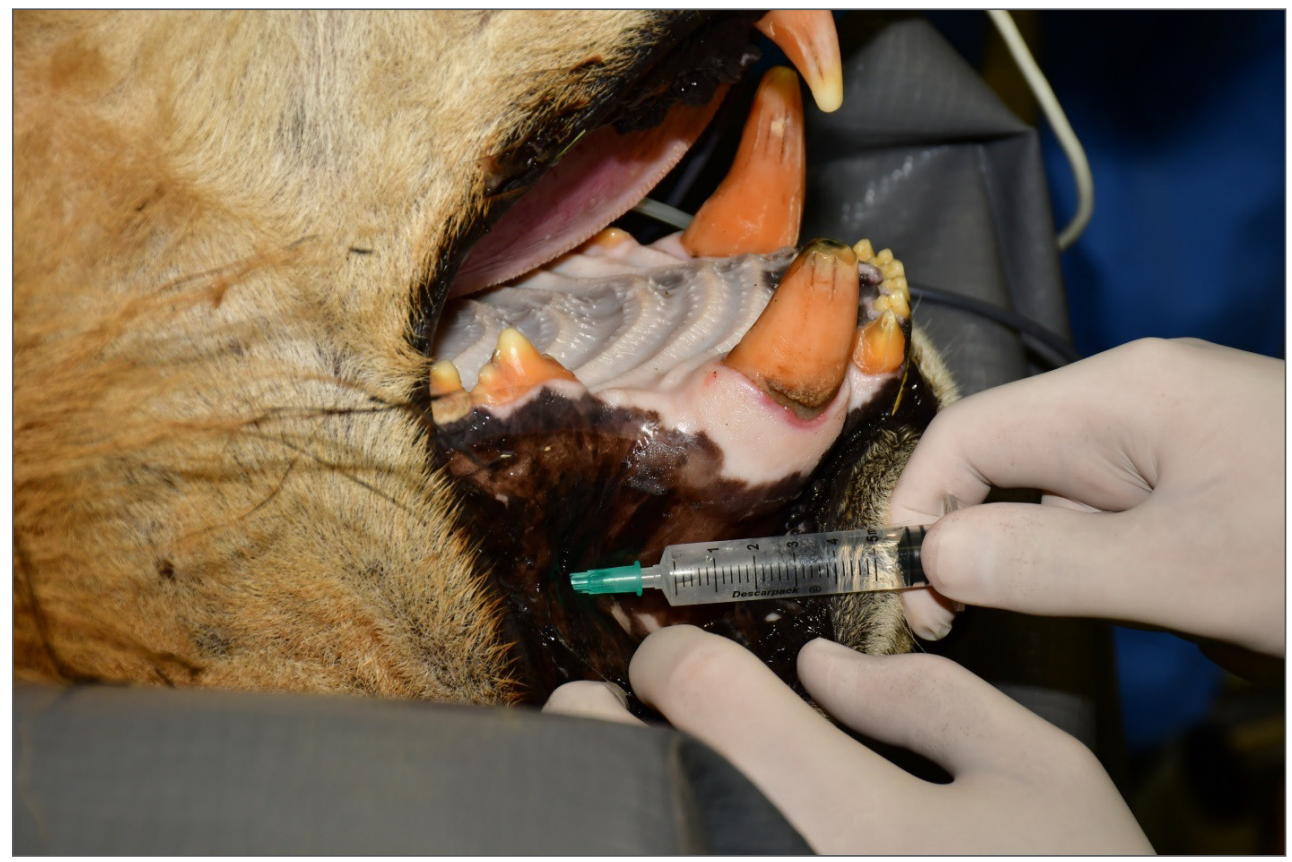

Fonte: os autores; fotografia cedida por Rogério Ribas Lange.

Além da venopunção da veia cefálica esquerda utilizada para a infusão dos fármacos, foi canulada a artéria metatársica esquerda para mensuração direta da pressão arterial pelo método invasivo. O leão foi monitorado por meio de monitor multiparamétrico veterinário Digicare LifeWindow
LW9xVet constantemente e os parâmetros foram registrados a cada cinco minutos, desde o período de indução (T0) até o final da infusão (T125), sendo avaliadas frequências cardíaca e respiratória, temperatura esofágica precordial e pressões arteriais sistólica, diastólica e média (Tabela 1).

Tabela 1 - Registro transanestésico das frequências cardíaca e respiratória, temperatura esofágica e pressões arteriais sistólica, média e diastólica, em minutos, do paciente Rawell (Panthera leo), submetido a tratamento endodôntico no Hospital Veterinário da Universidade Federal do Paraná (HV-UFPR).

\begin{tabular}{ccccccc}
\hline \multirow{2}{*}{$\begin{array}{c}\text { Tempo de } \\
\text { anestesia } \\
(\mathbf{m i n})\end{array}$} & $\begin{array}{c}\text { FC } \\
\mathbf{( b p m )}\end{array}$ & $\begin{array}{c}\text { FR } \\
(\mathbf{m p m})\end{array}$ & $\begin{array}{c}\text { TE } \\
\mathbf{(} \mathbf{(} \mathbf{C})\end{array}$ & $\begin{array}{c}\text { PAS } \\
(\mathbf{m m H g})\end{array}$ & $\begin{array}{c}\text { PAM } \\
(\mathbf{m m H g})\end{array}$ & $\begin{array}{c}\text { PAD } \\
(\mathbf{m m H g})\end{array}$ \\
\hline T0 & 139 & 12 & 36,2 & 119 & 90 & 76 \\
T5 & 67 & 18 & 36,3 & 119 & 90 & 76 \\
T10 & 67 & 13 & 36,7 & 136 & 108 & 94 \\
T15 & 68 & 6 & 36,9 & 136 & 108 & 94 \\
T20 & 64 & 6 & 37,1 & 138 & 119 & 109 \\
T25 & 71 & 11 & 37,1 & 136 & 108 & 94 \\
\end{tabular}


Continuação

$\begin{array}{ccccccc}\text { T30 } & 64 & 5 & 37,2 & 85 & 52 & 40 \\ \text { T35 } & 65 & 6 & 37,1 & 87 & 50 & 38 \\ \text { T40 } & 63 & 6 & 37,2 & 93 & 60 & 47 \\ \text { T45 } & 63 & 5 & 37,2 & 92 & 62 & 50 \\ \text { T50 } & 63 & 5 & 37,2 & 91 & 60 & 49 \\ \text { T55 } & 64 & 12 & 37,2 & 93 & 60 & 49 \\ \text { T60 } & 63 & 13 & 37,3 & 94 & 61 & 48 \\ \text { T65 } & 64 & 12 & 37,3 & 101 & 69 & 55 \\ \text { T70 } & 65 & 12 & 37,3 & 103 & 70 & 56 \\ \text { T75 } & 65 & 18 & 37,3 & 106 & 71 & 58 \\ \text { T80 } & 66 & 7 & 37,3 & 108 & 71 & 59 \\ \text { T85 } & 64 & 10 & 37,3 & 109 & 74 & 61 \\ \text { T90 } & 63 & 12 & 37,3 & 104 & 69 & 56 \\ \text { T95 } & 63 & 11 & 37,3 & 103 & 68 & 57 \\ \text { T100 } & 68 & 12 & 37,3 & 104 & 70 & 57 \\ \text { T105 } & 63 & 10 & 37,3 & 106 & 72 & 61 \\ \text { T110 } & 66 & 25 & 37,3 & 106 & 73 & 62 \\ \text { T115 } & 67 & 9 & 37,3 & 110 & 76 & 63 \\ \text { T120 } & 66 & 20 & 37,3 & 102 & 69 & 56 \\ \text { T125 } & 68 & 24 & 37,3 & 103 & 70 & 57\end{array}$

Legenda: T0 - imediatamente após indução (basal); T5 - cinco minutos após indução; T10 - dez minutos após indução [...]; T125 - cento e vinte e cinco minutos após a indução. FC - frequência cardíaca em batimentos por minuto; FR - frequência respiratória em movimentos por minuto; TE - temperatura esofágica em graus célsius; PAS - pressão arterial sistólica em milímetros de mercúrio; PAM - pressão arterial média em milímetros de mercúrio; PAD - pressão arterial diastólica em milímetros de mercúrio.

Fonte: os autores.

O procedimento anestésico teve duração de duas horas e 30 minutos e não foram observadas intercorrências anestésicas relacionadas aos sistemas orgânicos do animal. A ventilação mecânica foi interrompida 10 minutos antes do final do procedimento, permitindo a ventilação espontânea. Quando o paciente se manteve em eucapnia sem auxílio respiratório as infusões foram interrompidas e o paciente imediatamente levado para a caixa de contenção ainda intubado e inconsciente. A extubação foi realizada após nove minutos da interrupção das infusões, quando foram observados sinais de despertar anestésico caracterizados por presença de reflexos palpebrais e movimentações auricular e da língua. Após 55 minutos de anestesia o paciente apresentava sustentação espontânea da cabeça, quando ele foi novamente transportado ao zoológico para soltura no recinto. A ambulação foi considerada normal pelos observadores no zoológico seis horas após a anestesia.

\section{Discussão}

A possibilidade de transportar um leão cativo de um zoológico para tratamento em outro local sem a necessidade de prévia contenção farmacológica é interessante, diminuindo dessa maneira a quantidade de medicamentos, o tempo de anestesia e consequentemente o tempo de recuperação anestésica. Para isso, é imprescindível o condicionamento de 
animais de zoológico para entrar em caixas de transporte, como realizado nesse caso. Porém, deve-se entender que o transporte de um leão, mesmo que em condições adequadas, está associado com alterações fisiológicas em decorrência de estresse, com liberação de catecolaminas para ativar uma resposta de luta ou fuga, aumentando dessa forma o metabolismo celular e consequentemente o requerimento por anestésicos para captura e contenção. ${ }^{(9)}$

De qualquer forma, a administração intramuscular de anestésicos para a contenção foi realizada por meio de dardo e zarabatana. Caixas de contenção que permitam a administração direta dos fármacos podem reduzir substancialmente $\mathrm{o}$ desperdício de medicamentos, seja pelo maior requerimento relacionado ao estresse do tiro e movimentação subsequente, como pela possibilidade de erros de tiro ou falha do dardo. ${ }^{(10)}$ Situações de estresse, medo, excitação, dor ou qualquer outra condição quando os níveis de catecolaminas endógenas estão elevados podem tornar os animais refratários aos efeitos dos anestésicos administrados, especialmente agonistas de receptores $\alpha$ - 2 adrenérgicos como a dexmedetomidina, podendo haver necessidade do incremento das doses dos fármacos, já que haverá interferência na redução de liberação de neurotransmissores excitatórios induzida por eles. ${ }^{(11)}$ A combinação de tiletamina-zolazepam e de um opioide com um agonista do receptor $\alpha-2$ adrenérgico diminui o volume injetado, aumenta a analgesia e diminui o tempo de recuperação após a contenção. ${ }^{(12-17)}$

Além da adequada estabilidade hemodinâmica do paciente cirúrgico, a TIVA, quando comparada à anestesia inalatória, apresenta vantagens, como redução do estresse cirúrgico, menor depressão cardiovascular, independência do sistema respiratório para a absorção e transporte dos agentes anestésicos, menos alterações hormonais e ausência de poluição ambiental, não expondo o paciente e a equipe cirúrgica aos efeitos dos agentes inalatórios. ${ }^{(18-19)}$
Os efeitos indesejáveis mais comuns do propofol são hipotensão e depressão cardiorrespiratória dose-dependentes. ${ }^{(20-21)}$ Não foram observadas, entretanto, depressão respiratória devido à realização preventiva de ventilação mecânica logo após a indução da anestesia ou hipotensão, provavelmente devido à baixa taxa de infusão utilizada no procedimento. Apesar do volume corrente e da frequência respiratória terem sido pré-estabelecidos pelo operador, o animal começou a apresentar assincronia paciente-ventilador (APV), exigindo um reajuste constante da frequência respiratória ao longo do procedimento. A APV é um fenômeno comum e pode ocorrer devido a fatores relacionados ao paciente, ao ventilador ou a ambos. ${ }^{(22)}$

A associação da infusão contínua de remifentanil ao propofol pode reduzir o seu requerimento em mais de $40 \%$, diminuindo o tempo necessário para recuperação anestésica quando utilizado por período prolongado em gatos domésticos, minimizando os efeitos indesejáveis. ${ }^{(8)}$ Tal constatação pôde ser efetivamente sustentada nesse relato, ao observar que a taxa de infusão de propofol para manutenção utilizada neste leão acompanhado manteve-se entre 0,02 e $0,1 \mathrm{mg} / \mathrm{kg} / \mathrm{min}$, com sua associação à infusão contínua de dexmedetomidina e remifentanil.

O remifentanil é um fármaco derivado do fentanil com curta duração de ação e sem efeito cumulativo em doses repetidas ou em infusão contínua. Além de produzir analgesia potente, confere estabilidade hemodinâmica durante a manutenção anestésica, principalmente quando administrado por infusão contínua e, por isso, é o agente de escolha em procedimentos longos em felídeos. ${ }^{(8)} \mathrm{A}$ dexmedetomidina, por sua vez, é um fármaco altamente seletivo por receptores $\alpha-2$ adrenérgicos que oferece sedação, analgesia e relaxamento muscular, não produzindo depressão respiratória importante. ${ }^{(23-25)}$ Além desses efeitos, a dexmedetomidina produz alterações hemodinâmicas notáveis, como bradicardia e aumento da resistência vascular 
sistêmica. Como essas alterações frequentemente persistem durante a anestesia, uma das vantagens na utilização dos fármacos deste grupo está na possibilidade de reversão de seus efeitos por meio de antagonistas $\alpha-2$ adrenérgicos. ${ }^{(26)}$ Apesar de seus efeitos notáveis, ainda são poucos os relatos do emprego de dexmedetomidina e remifentanil em felídeos selvagens, e nenhum, a conhecimento dos autores deste manuscrito, que relate nesta espécie o uso de infusões contínuas desses fármacos no período transanestésico.

A temperatura corpórea de leões anestesiados com xilazina, cetamina e isofluorano ou então contidos com butorfanol, medetomidina e midazolam mostrou variação entre $36,6{ }^{\circ} \mathrm{C}$ e $40,3{ }^{\circ} \mathrm{C} .{ }^{(27-28)}$ A temperatura corpórea do paciente deste relato manteve-se ligeiramente inferior, entre $36,2{ }^{\circ} \mathrm{C}$ e $37,4{ }^{\circ} \mathrm{C}$. Essa diminuição da temperatura pode ser explicada pela redução da capacidade de termorregulação decorrente da ação dos fármacos anestésicos, além do clima frio no dia da anestesia, da longa duração do procedimento e da exposição do paciente durante o período de transporte. Não foi caracterizada, porém, hipotermia, que é genericamente caracterizada por uma temperatura corpórea abaixo de $35{ }^{\circ} \mathrm{C}$, quando podem ser observadas alterações como um maior tempo de recuperação, assim como predispor o animal à acidose, coagulopatias e $\operatorname{arritmias.}^{(29)}$

Foi observada alta frequência cardíaca, acima de 90 bpm, nos primeiros minutos após a indução anestésica, explicada pelo estresse do transporte e administração do dardo anestésico, além da ação taquicardizante da tiletamina. Além disso, a laringoscopia e a intubação endotraqueal podem estar associadas a uma alteração hemodinâmica transitória, caracterizada por hipertensão e taquicardia. $^{(30)} \mathrm{O}$ efeito é transitório, ocorrendo 30 segundos após o início e com duração inferior a 10 minutos, ${ }^{(31)}$ como o observado nesse relato. Após a indução, a frequência cardíaca manteve-se dentro dos parâmetros fisiológicos da espécie, entre 63 e
71 bpm, e mais estável que aquele observado na imobilização de leões africanos com butorfanol, medetomidina e midazolam. ${ }^{(28)}$

O tempo de extubação foi rápido, considerando a duração do procedimento, semelhante ao observado com gatos domésticos. ${ }^{(8)}$ Um completo despertar com deambulação normal pode ser acelerado pela reversão dos efeitos da dexmedetomidina com um antagonista $\alpha-2$ adrenérgico como o atipamezole. ${ }^{(15-16)}$ Considerando a necessidade de novo transporte do leão até o zoológico e sua soltura no recinto, um maior período de recuperação total mantendo o paciente ainda com efeitos sedativos é desejável. Mesmo que a reversão da dexmedetomidina fosse realizada, os efeitos de acumulação do propofol em tecidos periféricos de gatos promove um recuperação mais lenta e suave, interessante para o uso clínico em felídeos selvagens. ${ }^{(8)}$

\section{Conclusões}

O protocolo de TIVA neste leão africano mostrou-se eficaz e seguro. Nenhum efeito indesejável foi observado com as infusões de propofol, remifentanil e dexmedetomidina, destacando que os parâmetros fisiológicos avaliados não foram significativamente influenciados pelo protocolo proposto em 150 minutos de anestesia. O despertar anestésico foi rápido e a recuperação total foi suave e com duração moderada.

\section{Referências}

1 Santos F, Salas ER, Bicca-Marques JC, ClegaroMarques C, Farias, EMP. Cloridrato de tiletamina associado com cloridrato de zolazepam na tranquilização e anestesia de calitriquídeos (Mammalia, Primates). Arq Bras Med Vet Zootec. [Internet]. 1999 [citado 2021 abr 15]; 51(6):539-45. Disponível em: http://www.scielo. br/scielo.php?script=sci_arttext\&pid=S010209351999000600006\&lng=pt\&tlng=pt\#corresp 
2 Walter O. Management guidelines for the welfare of elephants: elephants Loxodonta africana and Elephas maximus. $3^{\text {rd }}$ ed. London: British \& Irish Association of Zoos \& Aquariums, Regent's Park; 2010.

3 Galante R. Anestesia intravenosa total em primatas: comparação da infusão contínua de propofol com bolus intravenosos de tiletamina e zolazepam e associação de propofol com opioides ou cetamina [dissertação]. Curitiba (PR): Universidade Federal do Paraná; 2013.

4 Ferro PC, Nunes N, Paula DP, Nishimori CT, Conceição EDV, Guerrero PNH, et al. Variáveis fisiológicas em cães submetidos à infusão contínua de diferentes doses de propofol. Ciênc Rural. 2005;35(5):1103-8. doi: https:// doi.org/10.1590/S0103-84782005000500018

5 Aoki M, Wakuno A, Kushiro A, Mae N, Kakizaki M, Nagata SI, et al. Evaluation of total intravenous anesthesia with propofolguaifenesin-medetomidine and alfaxaloneguaifenesin-medetomidine in Thoroughbred horses undergoing castration. J Vet Med Sci. 2017; 79(12):2011-18. doi: 10.1292/jvms.16-0658.

6 Camu F, Lauwers M, Vanlersberghe C. Anestesia venosa total. In: White PF. Tratado de anestesia venosa. Porto Alegre: Artmed; 2001. p. 563-77.

7 Kramer KJ, Ganzberg S, Prior S, Rashid RG. Comparison of propofol-remifentanil versus propofol-ketamine deep sedation for third molar surgery. Anesth Prog. 2012;59(3):10717. doi: 10.2344/12-00001.1.

8 Marks M. Infusão contínua de propofol e remifentanil por longo período em gatos [dissertação]. Curitiba (PR): Universidade Federal do Paraná; 2017.

9 Vilani RGOC. Anestesia injetável e inalatória. In: Cubas ZSC, Silva CR, Catão-Dias JL. Tratado de animais selvagens: medicina veterinária. 2a ed. São Paulo: Roca; 2014. p. 182663.

10 Ryeng KA, Arnemo JM, Larsen S. Determination of optimal immobilizing doses of a medetomidine hydrochloride and ketamine hydrochloride combination in captive reindeer.
Am J Vet Res. 2001;62(1):119-26. doi: 10. 2460/ajvr.2001.62.119.

11 Lemke KA. Anticholinergics and sedatives. In: Tranquilli WJ, Thurmon JC, Grimm KA. Lumb \& Jones: veterinary anesthesia and analgesia. $4^{\text {th }}$ ed. Ames: Blackwell Publishing; 2007. p. 203-39.

12 Cattet MRL, Caulkett NA, Polischuk SC, Ramsay MA. Anesthesia of polar bears (Ursus maritimus) with zolazepam-tiletamine, medetomidine-ketamine, and medetomidinezolazepam-tiletamine. J Zoo Wildl Med. [Internet]. 1999 [cited 2021 jan 15]; 30(3):35460. Available from: https://www.ncbi.nlm.nih. gov/pubmed/10572857

13 Cattet MRL, Caulkett NA, Lunn NJ. Anesthesia of polar bears using xylazine- zolazepamtiletamine or zolazepam-tiletamine. J Wildl Dis. 2003;39(3):655-64. doi: 10.7589/00903558-39.3.655.

14 Caulkett NA, Cattet MR, Caulkett JM, Polischuk SC. Comparative physiological effects of telazol, medetomidine-ketamine, and medetomidine-telazol in polar bears (Ursus maritimus). J Zoo Wildl Med. [Internet]. 1999 [cited 2021 Apr 15]; 30(4):504-9. Available from: https://www.ncbi.nlm.nih.gov/pubmed/ 10749435

15 Caulkett NA, Cribb PH, Haigh JC. Comparative cardiopulmonary effects of carfentanilxylazine and medetomidine-ketamine used for immobilization of mule deer and mule deer/ white-tailed deer hybrids. Can J Vet Res. [Internet]. 2000 [cited 2020 Apr 15]; 64(1):64-8. Available from: https://www.ncbi.nlm.nih.gov/ pubmed/10680659

16 Millspaugh JJ, Brundige GC, Jenks JA, Tyner CL, Hustead DR. Immobilization of rocky mountain elk with Telazol and xylazine hydrochloride, and antagonism by yohimbine hydrochloride. J Wildl Dis. 1995;31(2):259-62. doi: 10.7589/0090-3558-31.2.259.

17 Murray S, Ware L, Monfort SL, Ware L, McShea J, Bush M. Anesthesia in female whitetailed deer using Telazol and xylazine. J Wildl Dis. 2000;36(4):670-5. doi: 10.7589/00903558-36.4.670. 
18 Aguiar AJA. Anestesia intravenosa total. In: Fantoni DT, Cortopassi SRG. Anestesia em cães e gatos. $2^{a}$ ed. São Paulo: Roca; 2010. p. 275-97.

19 Augusto MM. Anestesia intravenosa total [dissertação]. Curitiba: Universidade Federal do Paraná; 2010.

20 Kotani Y, Shimazawa M, Yoshimura S, Iwama T, Hara H. The experimental and clinical pharmacology of propofol, an anesthetic agent with neuroprotective properties. CNS Neurosci Ther. 2008;14(2):95-106. doi: 10.1111/j.15273458.2008.00043.x.

21 Paula DP, Nunes N, Nishimori CTD, Lopes PCF, Carareto R, Santos PSP. Efeitos da infusão contínua de propofol ou etomidato sobre variáveis intracranianas em cães. Arq Bras Med Vet Zootec. 2010;62(2):302-8. https://doi. org/10.1590/S0102-09352010000200009

22 Holanda MA, Vasconcelos RS, Ferreira JC, Pinheiro BV. Assincronia paciente-ventilador. J Bras Pneumol. [Internet]. 2018 [citado 2021 abr 15]; 44(4):321-33. Disponível em: http://www.scielo.br/scielo.php?script=sci arttext\&pid=S1806-37132018000400321\&1n $\mathrm{g}=$ en\&tlng=en

23 Bagatini A, Gomes CR, Masella MZ, Rezer G. Dexmedetomidina: farmacologia e uso clínico. Rev Bras Anestesiol. [Internet]. 2002 [citado 2021 abr 15]; 52(5):606-17. Disponível em: http://www.scielo.br/pdf/rba/v52n5/v52n5a12. pdf

24 Plumb DC. Plumb's veterinary drugs handbook. $5^{\text {th }}$ ed. Cambridge: Blackwell Publishing; 2005 .

25 Cortopassi SRG, Fantoni, DT. Medicação préanestésica. In: Fantoni DT, Cortopassi SRG. Anestesia em cães e gatos. $2^{\mathrm{a}}$ ed. São Paulo: Roca; 2010. p. 217-27.

26 Martin-Flores M, Sakai DM, Honkavaara J, Campoy L, Portela DA, Gleed RD. Hemodynamic effects of MK-467 following intravenous administration to isoflurane-anesthetized cats concurrently receiving dexmedetomidine. Am J Vet Res. 2018;79(7):711-7. doi: 10.2460/ ajvr.79.7.711.
27 Bharathidasan M, William BJ, Jayaprakash R, Kannan TA, Thirumurugan R, George RS. Immobilization and anaesthesia in Asiatic lions (Panthera leo persica). Adv Anim Vet Sci. 2016;4(3):134-44. doi: 10.2139/ssrn.3524484.

28 Wenger S. Buss P, Joubert J, Steenkamp J, Shikwambana P, Hatt JM. Evaluation of butorphanol, medetomidine and midazolam as a reversible narcotic combination in free-ranging African lions (Panthera leo). Vet Anaesth Analg. 2010;37(6):491-500. doi: 10.1111/j.1467-2995. 2010.00569.x.

29 Caulkett NA, Arnemo JM. Comparative anesthesia and analgesia of zoo animals and wildlife. In: Grimm KA, Lamont LA, Tranquilli WJ, Greene SA, Robertet SA, editors. Lumb \& Jones: veterinary anesthesia and analgesia. $5^{\text {th }}$ ed. New Jersey: John Wiley \& Sons, Inc.; 2015. p. 764-76.

30 Dhanya PR, Franklin D. Effect of oral Pregabalin on hemodynamic stress response to laryngoscopy and endotracheal intubation. J Med Sci Clin Res. 2017;5(4):20678-84. doi: https:// dx.doi.org/10.18535/jmscr/v5i4.153

31 Gupta A, Wakhloo R, Gupta V, Kapoor B. Comparison of esmolol and Lignocaine for attenuation of cardiovascular stress response to laryngoscopy and endotracheal intubation. JK Sci. [Internet]. 2009 [cited 2021 Apr 15]; 11(2):78-81. Available from: http://www. jkscience.org/archive/vol112/7\%20\%20 Original\%20Article-esmolol.pdf 